\title{
Design and Analysis of a Wire-driven Flexible Manipulator for Bronchoscopic Interventions
}

\author{
Ning Liu ${ }^{1}$, Christos Bergeles ${ }^{2}$, Guang-Zhong Yang ${ }^{1}$, Fellow, IEEE
}

\begin{abstract}
Bronchoscopic interventions are widely performed for the diagnosis and treatment of lung diseases. However, for most endobronchial devices, the lack of a bendable tip restricts their access ability to get into distal bronchi with complex bifurcations. This paper presents the design of a new wire-driven continuum manipulator to help guide these devices. The proposed manipulator is built by assembling miniaturized blocks that are featured with interlocking circular joints. It has the capability of maintaining its integrity when the lengths of actuation wires change due to the shaft flex. It allows the existence of a relatively large central cavity to pass through other instruments and enables two rotational degrees of freedom. All these features make it suitable for procedures where tubular anatomies are involved and the flexible shafts have to be considerably bent in usage, just like bronchoscopic interventions. A kinematic model is built to estimate the relationship between the translations of actuation wires and the manipulator tip position. A scale-up model is produced for evaluation experiments and the results validate the performance of the proposed mechanism.
\end{abstract}

\section{INTRODUCTION}

Lung diseases, such as chronic obstructive pulmonary disease (COPD), and lung cancer, have attracted much attention due to their rising threats to human lives. According to statistics provided by the International Agency for Research on Cancer (IARC), lung and bronchus cancer has become the most common cause of cancer death [1]. Bronchoscopic interventions are effective procedures for the diagnosis and treatment of many lung diseases, and are preferred over open procedures for patient's benefits, with less complication, preserving airway integrity, and promoting faster recovery. During the intervention, a bronchoscope is inserted into the trachea or bronchi through the mouth or nose to visualize the inside situations of airways. The practitioner then passes specialized devices, which usually consist of a working distal end (e.g. forceps) and a long flexible shaft, through the instrument channel of the bronchoscope to perform specific procedures, for example, placing stents and retrieving specimen.

Due to the dimension limit, a bronchoscope is usually only able to access into the 3rd or 4th generations of the respiratory tree. A major problem would appear when the target area is out of the reach. For example, transbronchial biopsy is to collect tissue samples from peripheral bronchioles, the flexible forceps have to be guided to areas where the bronchoscope cannot approach directly. These forceps, unlike the

\footnotetext{
${ }^{1}$ Ning Liu and Guang-Zhong Yang are with the Hamlyn Centre for Robotic Surgery, Imperial College London, SW7 2AZ, London, UK (email: ning.liul3eimperial.ac.uk)

${ }^{2}$ Christos Bergeles is with the Department of Medical Physics and Bioengineering, University College London, NW1 2HE, London, UK
}

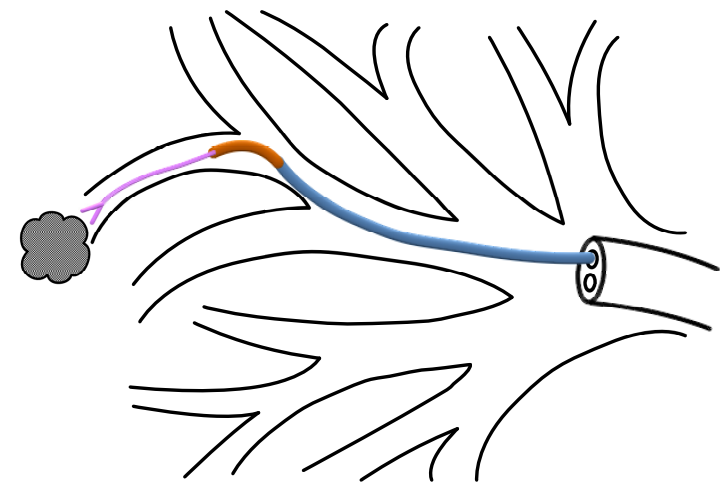

Fig. 1. The application of a flexible guiding device in transbronchial biopsy. The orange part denotes a bendable manipulator, it can help a regular bronchoscopic instrument get into bronchi at difficult bifurcations.

bronchoscope having a bendable distal end, cannot actively change its orientation during the advancement, which makes it extremely difficult to get into bronchi with acute bending angles.

As demonstrated in Fig. 1, a solution to this problem is to take advantage of a flexible tubular guiding device that is able to fit into the working channel of a regular bronchoscope while leaving enough central cavity to let other devices pass through. This paper focuses on the development of a bendable continuum manipulator. The proposed manipulator takes advantage of a unique design of building blocks with interlocking circular joints and is suitable for occasions where a flexible shaft needs to be employed.

The rest of the paper is organized as follows: Section II introduces existing continuum manipulators, Section III describes the challenges in robot design for bronchoscopic interventions and our proposed manipulator, Section IV characterizes the new wire-driven mechanism with its kinematics model and workspace, Section V presents the setup and results of the validation experiment, Section VI concludes the findings of this work and discusses the future work at the next phase.

\section{EXISTING DESIGNS OF CONTINUUM MANIPULATORS}

Continuum manipulators have made significant inroads towards in vivo applications in recent years, together with the ongoing evolution of minimally invasive surgery (MIS). They help instruments like endoscopes to overcome obstacles, follow curved pathways, and reach anatomical targets where no direct access exists. Popular continuum robots can be classified into four types by their actuation mechanism. The 
first kind uses soft structures to facilitate flexibility and is usually driven by compressed air. For example, McMahan et al. built the OctArm manipulator driven by air muscle actuators [2]. However, they are not suitable for MIS owing to the cumbersome size and inherent difficulty in precision control. The second type is the serpentine robot, which consists of multiple rigid links driven by motors. It has advantages over other types like more degrees of freedom (DoF) and higher control precision, and is promising in MIS with large lumen. Shang et al. built an 7-DoF articulated robot with embedded micro motors [3]. Its outer diameter is only $12.5 \mathrm{~mm}$ and could perform a natural orifice translumenal endoscopic surgery (NOTES) tubal ligation procedure. The main issues associated with this mechanism falls on the difficulty in miniaturization and complexity of robot control. The third classification involves pre-curved concentric tubes [4]. They have proper sizes for MIS but might have high risks of material yielding especially when they have to follow the complex paths like human airways

The wire-driven mechanism, counted as the fourth type, is the most widely used in MIS tasks because of the potential for miniaturization and ease of control and manufacturing. The basic principle in the design of wire-driven robot requires a flexible vertebra to support the continuum body, and antagonistic tendons running outside at equal separation to control the body configurations. Amounts of relevant studies have been done [5]-[10], and two categories can be found from these designs.

- The first class of wire-driven continuum robot takes advantage of material flexure. A popular example consists of a flexible backbone in the middle with cable-guide disks fastened to it [6], [7]. However, this design lacks a working channel that is required by our platform. Other variations work by selecting elastic tubes and cutting parallel notches to facilitate tube bending [8]. The major concern is metal fatigue, especially for those near the notches. Besides, such design is more appropriate for 1-DoF tasks. Their capabilities of resisting axial forces would be degraded when adapted for 2-DoF applications.

- The other kind forms the continuum structure by assembling separate disks instead of using a continuum body directly. Li et al. proposed to use spherical joints to connect rigid links and built a multi-section snake-like robot [5]. Kim et al. proposed the neutral-line mechanism that strings disks with cylindrical surfaces, which is capable of changing its own stiffness by adjusting the tension of wires [10]. Its simple geometry also facilitates miniaturization. He et al., following this mechanism, made a flexible manipulator for retinal surgeries with a diameter of only $0.9 \mathrm{~mm}$ [9].

\section{MANIPULATOR WITH INTERLOCKING BLOCKS}

\section{A. Design Challenges}

Bronchoscopic interventions have stringent requires on the manipulator's dimensions and flexibility. On the basis
TABLE I

Manipulator Design SPECIFICATIONS

\begin{tabular}{|l||l|}
\hline Outer diameter & $\leq 2 \mathrm{~mm}$ \\
\hline Working channel diameter & $\geq 1.2 \mathrm{~mm}$ \\
\hline Length & $\approx 20 \mathrm{~mm}$ \\
\hline Degree of freedom & $2 \mathrm{DoF}$ rotation \\
\hline Range of motion & each DoF $\pm \geq 65^{\circ}$ \\
\hline
\end{tabular}
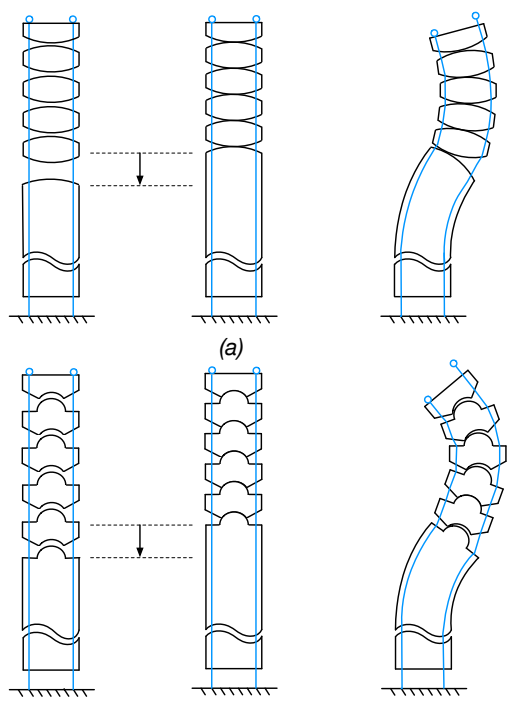

(b)

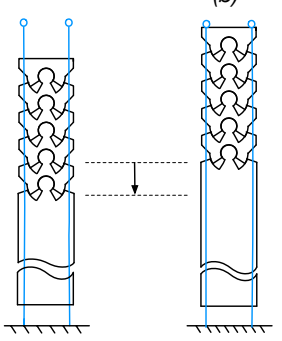

(c)

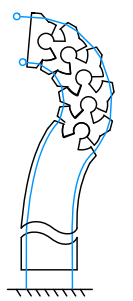

Fig. 2. Comparison of manipulators with (a) the neutral-line mechanism (curved surfaces), (b) speherical joints, and (c) proposed interlocking joints when their flexible shafts are compressed (left), kept straight (middle), and bent(right). This figure only shows $1-\mathrm{DoF}$ situations.

of our investigations on existing commercial bronchoscopes and interviews with physicians in Royal Brompton Hospital (London, UK), the outer diameter of the manipulator is suggested to be no bigger than $2 \mathrm{~mm}$ so that it can fit into most bronchoscopes' channels. A central lumen with a minimal diameter of $1.2 \mathrm{~mm}$ is also demanded to pass standard bronchoscopic instruments. The length of this dexterous unit should be around $20 \mathrm{~mm}$ to facilitate clinical applications. Given there is a limit of $60^{\circ}$ for most airway branching angles [11] and it is difficult to rotate the manipulator inside airways, two rotational DoFs with at least $\pm 65^{\circ}$ range of bending for each DoF are preferred. The design specifications are summarized in Table I.

Apart from the above physical restrictions, bronchoscopic interventions also pose challenges on the wire-driven mech- 
anism. In order to extend the range of workspace, the dexterous manipulator is usually connected to a shaft which contains and guides wires to the actuation pack. On most occasions, this shaft is rigid or only with very limited flexibility, therefore the lengths of wires inside the shaft experience little change during operation. However, for bronchoscopic interventions, the shaft is very flexible and has to be considerably bent when approaching target bronchioles, especially for those in superior lobes. The shaft flex would lead to the length inequality of antagonistic wires inside the shaft and force the distal end to bend. This uncontrollable bending could possibly permanently damage flexure-based manipulators. For those assembled from separate joints, the wire length changes could influence the wire tension and affects their control. As shown in Fig. 2, the assumptions about the kinematics modelling of the neutral-line mechanism do not hold when the shaft deflects a big angle, because the tension in the right wire is obviously reduced. For spherical joints, without the compression of right wire, the joint connections become loose and the motions of links no longer comply with expected geometric rules. Moreover, typical flexible shafts use wound metal strips or coils, they can be stretched and then compressed when pulmonologists pull and push the proximal ends. The sudden length reduction of wires inside the shaft could make the continuum manipulator temporarily disjoint, which should be avoided in procedures because of the risk of tissue damage.

\section{B. Design}

The proposed manipulator emphasizes miniaturization, 2DoF flexibility, and the ability to cope with shaft flex. Like presented in Fig. 3(a), the basic building blocks are identical disk-like elements that incorporates interlocking joints. They should be manufactured by selecting a metal tube and cutting two complementary patterns from orthogonal directions. The joints are circular and work like standard revolute joints, even though their motions are not independent. The whole robot can be assembled by aligning the elements alternately in two orthogonal directions (See Fig. 3(b)). In case the tendons will interfere with the pivoting of the joint, a notch is cut off from the upper middle position of the joint circle.

As suggested in Table I, each block is $2 \mathrm{~mm}$ in outer diameter and has a central cavity with $1.2 \mathrm{~mm}$ diameter. The four pilots holes around the central lumen are $0.2 \mathrm{~mm}$ in diameter and used to guide actuation wires, whose one ends are stuck to the manipulator's distal tip by ball head pins, $p_{1}, p_{2}$ and $t_{1}, t_{2}$ indicate two pairs of antagonistic wires. The axial distance between adjacent orthogonal joints' centres can be as small as $1 \mathrm{~mm}$, which means the whole manipulator can hold 20 blocks at most. For each joint, the biggest pivoting angle is $15^{\circ}$, therefore a maximal bending angle of $150^{\circ}$ for each DoF can be achieved.

The bending of the manipulator relies on wire length changes. When $p_{1}$ contracts and $p_{2}$ extends, an axial force and a moment is applied on the manipulator and drives the manipulator to bend. Fig. 3(c) illustrates the manipulator in a bending position. A common drawback with the wire-driven
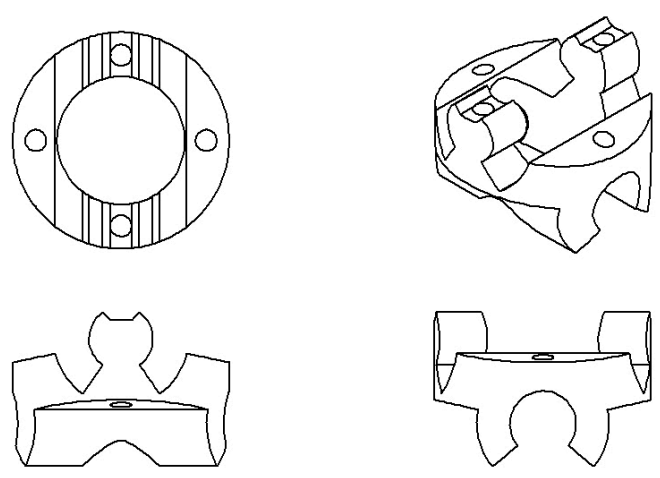

(a)
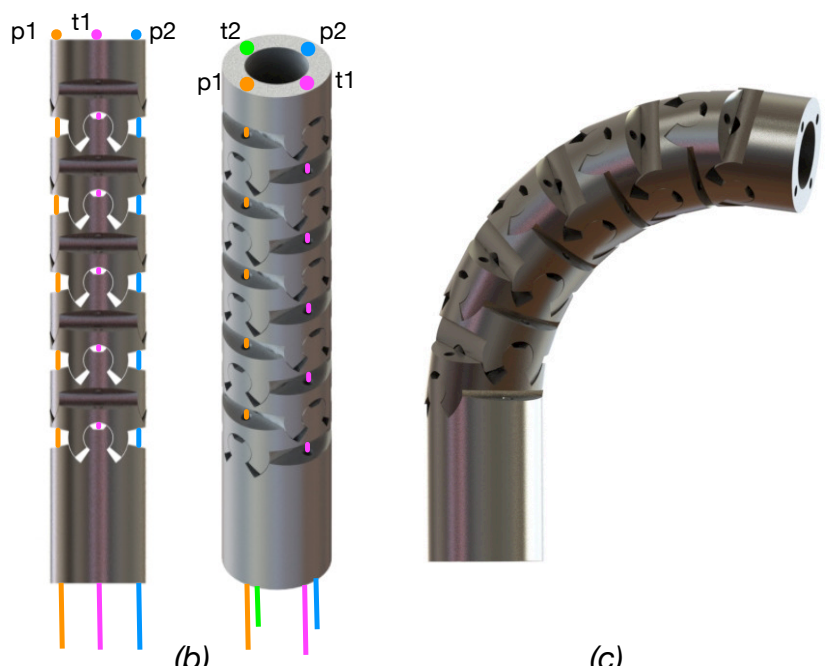

(b)

(c)

Fig. 3. Continuum flexible manipulator design: (a) 2D engieering drawings of the basic building blocks, (b) the manipulator assembly in the straight configuration, (c) the manipulator in a bending posture. Colourful lines in (b) represents actutation wires, but they are not outlined in (c).

mechanism like the proposed design, whose length invariant neutral line is located in the middle, is that the manipulator can have various configurations with the same wire length changes [10]. In fact, according to our preliminary experiments in which non-elastic fishing lines were used to drive the robot, the tubular arm would hardly keep straight when the motors pulled it back from a bending position. This might result from the integral effect of frictions. Therefore the super-elastic nitinol wires are suggested in this application, they could serve as the flexible backbone, counteract the disturbance of the friction, guarantee the manipulator is straight when no wires contract, help keep positioning stable and improve robot control. We can also use a flexible rubber tube to strengthen this backbone.

The interlocking revolute joints can not only simplify the motion modelling but also improve the manipulator's ability to cope with shaft flex. Fig. 2(c) depicts the occasions when the flexible shaft is compressed or deflected. In both situations, the manipulator can maintain its integrity and comply with expected geometric rules. 


\section{AnAlytical CharaCteris ation}

A. Basic relationship between wire length changes and joint bending angles

Like an articulated robot, the joints are the fundamental elements when modelling the proposed mechanism. The differences, however, are due to the fact that the joints of the manipulator are not independent. We would divide all joints into two groups, those controlled by $p_{1}, p_{2}$ are called $\mathbf{p}$ joints, and those mainly influenced by $t_{1}, t_{2}$ are $\mathbf{t}$ joints. The pivoting angles are identical for joints in the same group, which are $\theta_{p}$ and $\theta_{t}$ respectively. The numbers of $\mathbf{p}$ and $\mathbf{t}$ joints are equal, denoted as $N$.

With the assumption of no external loads, the relationship between the joint bending angles and the wire translations can be derived from the joint geometry. There are some variables in Fig. 4 needs to be explained at first. The green circles denote the rotation centres. $R_{j}$ connects the rotation centre and the guide holes on left or right side. $R_{d}$ represents the distance between the guide-channel and tube middle axis. $h_{t 1}$ and $h_{t 2}$ are the gap distances beside joint $\mathbf{t}, h_{p 1}$ and $h_{p 2}$ are the gap distances beside joint p. $r_{1}$ and $r_{2}$ correspond to the radius of circular joints and the distance of rotation centre to the joint notch respectively. In the case of the proposed 2-DoF manipulator, the motions of two pairs of wires can affect each other. When a $\mathbf{p}$ joint is deflected by wire $p_{1}$ and $p_{2}$, the parts of wire $t_{1}$ and $t_{2}$ placed at the centre of this joint are also pulled (See Fig. 4 (b)). $h_{t 1}^{p}$ reflects the part of wire $t_{1}$ influenced by joint $\mathbf{p}$. Therefore,

$$
\Delta l_{t 1}=N\left(\Delta h_{t 1}+\Delta h_{t 1}^{p}\right)
$$

where $l_{t 1}$ indicates the total length change of wire $t_{1}$.

By observing Fig. 4 (a), we can get

$$
\begin{gathered}
h_{t 1}=2 R_{j} \sin \left(\frac{\alpha-\theta_{t}}{2}\right) \\
\Delta h_{t 1}=\frac{d}{d \theta_{t}} h_{t 1} \cdot \theta_{t}=-R_{j} \cos \left(\frac{\alpha-\theta_{t}}{2}\right) \theta_{t}
\end{gathered}
$$

Since $\theta_{t}$ is small $\left(<15^{\circ}\right)$,

$$
\Delta h_{t 1} \approx-R_{d} \theta_{t}
$$

From Fig. 4 (b), it can be found that

$$
\begin{gathered}
h_{t 1}^{p}=\sqrt{r_{1}^{2}+r_{2}^{2}-2 r_{1} r_{2} \cos \theta_{p}} \\
\Delta h_{t 1}^{p}=\frac{d}{d \theta_{p}} h_{t 1}^{p} \cdot \theta_{p}=\frac{r_{1} r_{2} \sin \theta_{p}}{\sqrt{r_{1}^{2}+r_{2}^{2}-2 r_{1} r_{2} \cos \theta_{p}}} \approx \frac{r_{1} r_{2}}{r_{1}-r_{2}} \theta_{p}
\end{gathered}
$$

Therefore,

$$
\Delta l_{t 1}=N\left(\Delta h_{t 1}+\Delta h_{t 1}^{p}\right) \approx N\left(-R_{d} \theta_{t}+\frac{r_{1} r_{2}}{r_{1}-r_{2}} \theta_{p}\right)
$$

Similarly, we can get the relations for other joints and wires,

$$
\begin{aligned}
& \Delta l_{t 2} \approx N\left(R_{d} \theta_{t}+\frac{r_{1} r_{2}}{r_{1}-r_{2}} \theta_{p}\right) \\
& \Delta l_{p 1} \approx N\left(-R_{d} \theta_{p}+\frac{r_{1} r_{2}}{r_{1}-r_{2}} \theta_{t}\right) \\
& \Delta l_{p 2} \approx N\left(R_{d} \theta_{p}+\frac{r_{1} r_{2}}{r_{1}-r_{2}} \theta_{t}\right)
\end{aligned}
$$

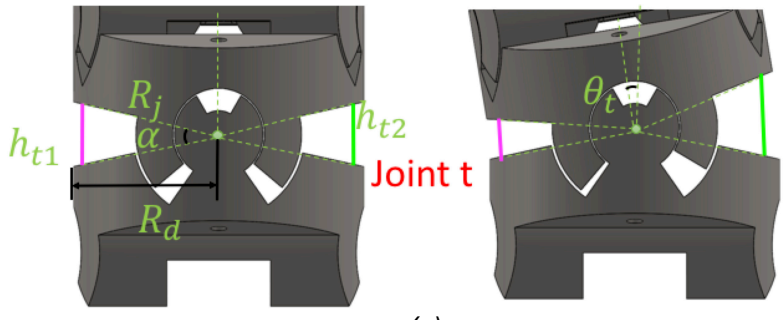

(a)

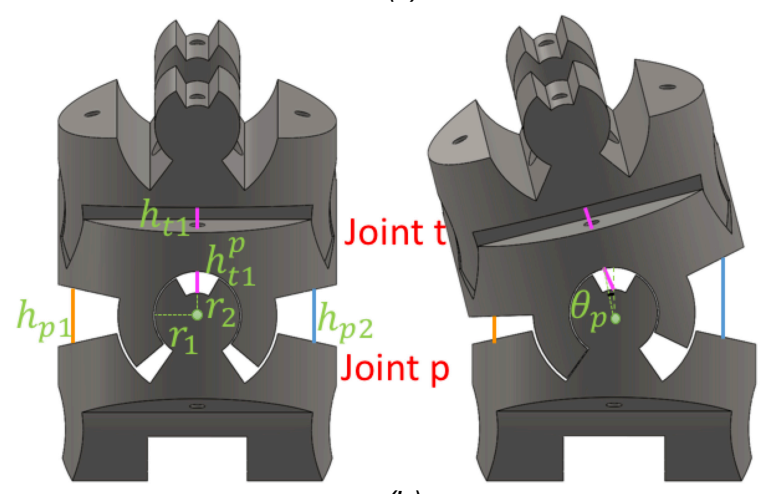

(b)

Fig. 4. Joint bending and corresponding wire length changes.

With all above equations, we can calculate the values of $\theta_{t}$ and $\theta_{p}$ by using

$$
\begin{gathered}
\theta_{t} \approx \frac{\Delta l_{t 2}-\Delta l_{t 1}}{2 N R_{d}} \\
\theta_{p} \approx \frac{\Delta l_{p 2}-\Delta l_{p 1}}{2 N R_{d}}
\end{gathered}
$$

\section{B. Kinematics and Workspace}

As suggested in [5], the kinematics can be divided into two parts (See Fig. 5): the mapping between the actuator space (i.e. lengths of wires) and manipulator's configuration space (i.e. $\theta_{p}$ and $\theta_{t}$ ), and the mapping between configuration space and task space (i.e. distal tip position). The former mapping is defined by forward kinematics $f_{1}$ and inverse kinematics $f_{1}^{-1}$, which has just been clarified in last subsection. The latter one, defined by $f_{2}$ and $f_{2}^{-1}$, will be explained in this section.

Since the joints of our proposed manipulator are standard revolute joints, the forward kinematics could be solved using Denavit-Hartenberg (D-H) method [12]. A gradientbased optimisation solver (fmincon, MATLAB) is adopted to calculate the inverse kinematics. It is capable of finding the optimum $\theta_{p}$ and $\theta_{t}$ values that minimise the distance between the real target point and the distal tip position produced by forward kinematics under non-linear constraints.

In terms of workspace, it should be a curved surface for this 2-DoF manipulator. However, analytical solutions are difficult to derive and it was solved in this work by sampling tip locations from forward kinematics and reconstruct the workspace surface by thin plate splines [13] (See Fig. $6)$. Then we can have a mathematical expression for the workspace, with which the controller is able to calculate 


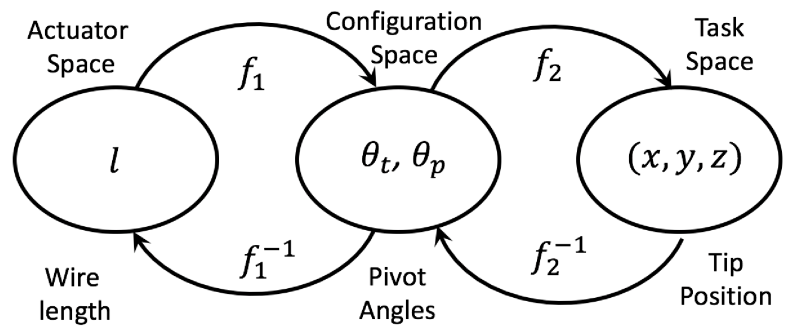

Fig. 5. Kinematics defined by mapping between actuator,configuration, and task space.

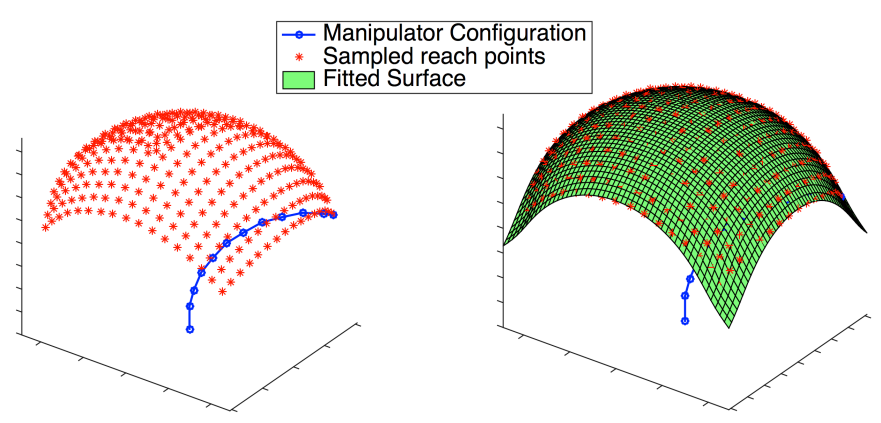

(a)

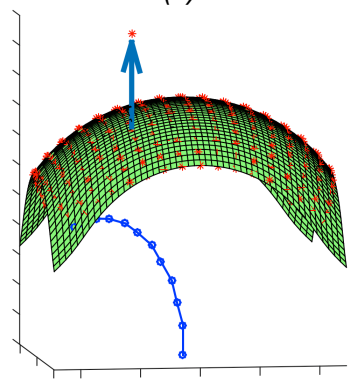

(b)

Fig. 6. The workspace of our 2-DoF manipulator. (a) The surface is constructed from sampled points using thin plate splines. (b)The blue arrow indicates the required translation displacement to locate the target point on the workspace surface.

the axial distance between the surface and any target point in task space. The practitioner could then pull or push the proximal end to make the target located in the manipulator's workspace and bend the distal end according to calculated inverse kinematics results.

\section{EXPERIMENTS AND RESULTS}

A 5:1 scale-up prototype was made for evaluation. The Objet260 3D printer was used to produce 11 building blocks made of VeroWhite. The prototype consists of five $\mathbf{p}$ joints and five $\mathbf{t}$ joints, allowing a maximal bending of $75^{\circ}$ for each DoF theoretically. The whole manipulator is $10 \mathrm{~mm}$ in outer diameter and $51.5 \mathrm{~mm}$ in length. A thin needle structure was mounted on the distal end to facilitate tracking. Four nitinol wires of $0.25 \mathrm{~mm}$ in diameter were employed for support and actuation. The experiment setup can be found in Fig. 7. Redundant actuations for every single wire were not implemented in this experiment. Instead, we used two

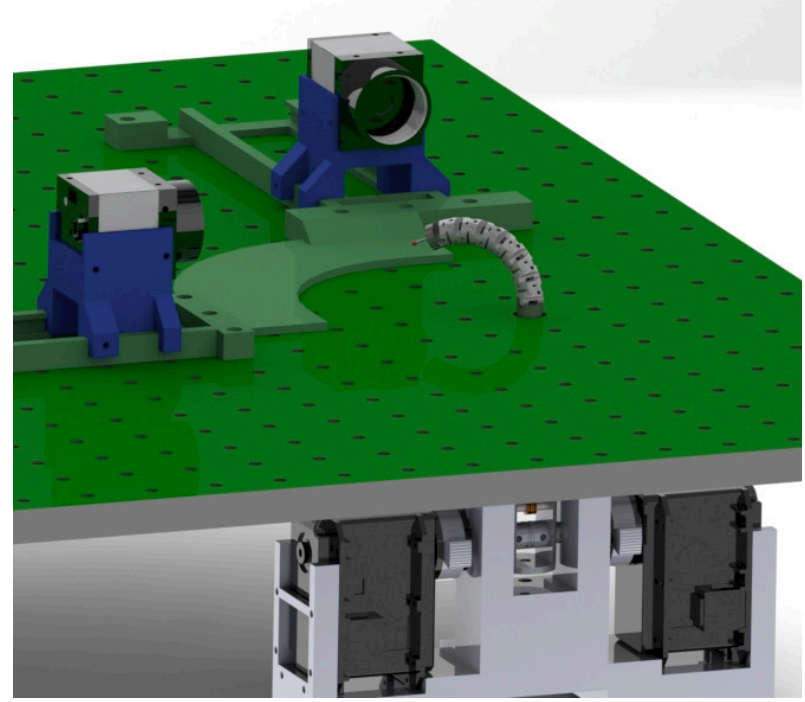

Fig. 7. Experiment Setup.

Dynamixel servo motors (ROBOTOIS, Korea) to control the translations of antagonistic wires. A two-camera system was used to take photos from orthogonal directions and track the motions of the manipulator. To enhance tracking robustness, the needle tip was painted red to contrast the green background. The experiments validated the kinematics modelling at two different levels: 1-DoF bending test and 2-DoF positioning test.

Only one pair of wires was actuated during the 1-DoF bending test. Seventy points on the manipulator's motion trajectory were recorded by cameras and corresponding tip bending angles at different positions were calculated (See Fig. 8 (a)). This experiment aimed to examine the relationship between the bending angles of the scale-up model and the translations of actuation wires. A comparison of the analytical predictions and the measured results is shown in Fig. 8. The captured data points demonstrate good linearity, which can be a proof of the previous kinematics analysis. The angle between two fitted lines is less than $1.3^{\circ}$. A possible source of such errors about line slope could be the inaccurate parameters measurements in equations (11) and (12). Even though this slope difference is small, the angle differences between the theoretical and experimental results do increase as the wire translation grows. Therefore a proper calibration would be necessary for future devices based on this design.

In terms of the 2-DoF test, we tracked the tip positions when the two pairs of wires are in various configurations. Since the two cameras can only cover a limited range of view, the manipulator was only bent in one quarter of its workspace, where it can be clearly captured by both cameras. A total of forty-nine tip points were measured and compared with the theoretical positions derived from the kinematics model, results are displayed in Fig. 9. All the points are nearly located on the same surface as expected. Similar to those results in the 1-DoF test, the positioning errors increase together with the wire translations, whose maximal value can 


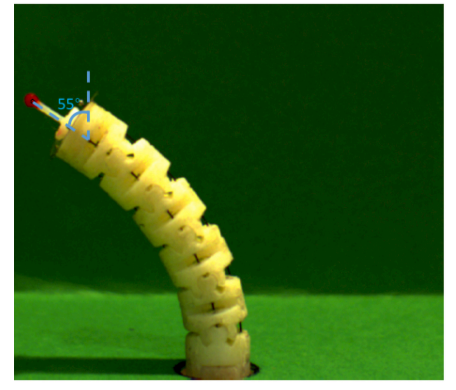

(a)

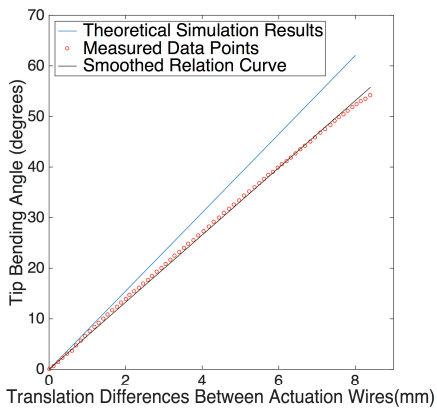

(b)
Fig. 8. The 1-DoF bending test. (a) the scale-up model is in a bending position of $55^{\circ}$, (b) the relation of the manipulator's bending angle with respect to the wire translation.

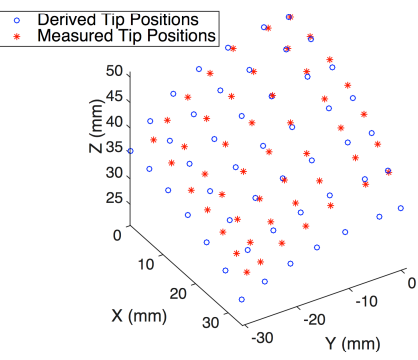

(a)

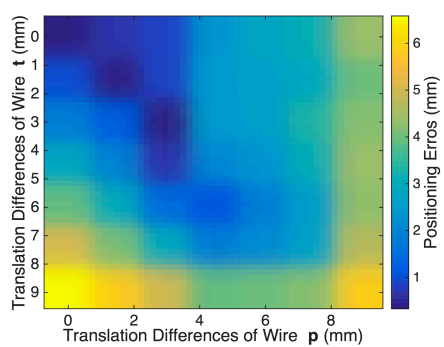

(b)
Fig. 9. The 2-DoF positioning test. (a) the theoretical positions derived from kinematics model vs the measured results, (b) the positioning errors heatmap with respect to the translations of two pairs of wires.

reach $6.58 \mathrm{~mm}$.

Apart from the inaccuracy of equation parameter estimation and variances during the manufacturing, there are also several other factors playing roles in disturbing the kinematics analysis, like the gaps around the circular joints and influences of all kinds of forces. The movements of individual blocks are combined actions of wire strains, wire elasticity, joint frictions, applied forces from adjacent blocks and blocks' own weights. The discrepancy could happen when wires are not strong enough to compensate the disturbances of other factors. For example, when wire strains deviate from the gravity direction, the latter will cause blocks fall away from their desired positions. This is more obvious on the distal blocks, where the deviation angles are big and the wire elasticity can no longer offset their weight effects. More detailed force analysis or a numerical model based on more comprehensive test data might be needed to reduce kinematics errors.

\section{CONCLUSION AND FUTURE WORK}

The design of a new continuum flexible manipulator is introduced to facilitate bronchoscopic interventions. It is built by assembling miniaturized blocks, which are featured with interlocking revolute joints. The proposed manipulator could work with flexible shafts and keep its own integrity even when the shaft is considerably deflected or compressed. The validation experiments demonstrate good linearity with regard to the relationship between wire translation and manipulator bending. However, more mechanics analysis still needs to be conducted to characterize its performance under external loads.

A real manipulator made of stainless steel and with desired dimensions is going to be manufactured by using high precision laser or wire cutting technology. The plan for the next phase would focus on the development of a robotic platform that combines the proposed manipulator, a flexible shaft and a hand-held control device. The control device will be equipped with four independent servo motors for actuation. Advanced control algorithms are being studied, which can adaptively compensate the wire length changes due to the shaft deformation. This should significantly improve the controllability of the manipulator in real procedures.

\section{ACKNOWLEDGMENT}

The authors would like to thank Dr. Christopher J. Payne and Dr. Jianzhong Shang for helpful discussions on the design and manufacturing of the actuation pack, and $\mathrm{Mr}$. Petros Giataganas and Mr. Konrad Leibrandt for their kind assistance with the camera rig.

\section{REFERENCES}

[1] J. Ferlay et al., "Cancer incidence and mortality worldwide: Iarc cancerbase no. 11 [Internet]," GLOBOCAN 2012 v1.0, Lyon, France: International Agency for Research on Cancer; 2013. Available from: http://globocan.iarc.fr, accessed on 2015/02/20.

[2] W. McMahan et al., "Field trials and testing of the octarm continuum manipulator," in Robotics and Automation, 2006. ICRA 2006. Proceedings 2006 IEEE International Conference on. IEEE, 2006, pp. 2336-2341.

[3] J. Shang et al., "An articulated universal joint based flexible access robot for minimally invasive surgery," in Robotics and Automation (ICRA), 2011 IEEE International Conference on. IEEE, 2011, pp. 1147-1152.

[4] P. E. Dupont et al., "Design and control of concentric-tube robots," Robotics, IEEE Transactions on, vol. 26, no. 2, pp. 209-225, 2010.

[5] Z. Li and R. Du, "Design and analysis of a bio-inspired wire-driven multi-section flexible robot," Int J Adv Robotic Sy, vol. 10, no. 209, 2013

[6] K. Xu and N. Simaan, "Analytic formulation for kinematics, statics, and shape restoration of multibackbone continuum robots via elliptic integrals," Journal of Mechanisms and Robotics, vol. 2, no. 1, p. 011006, 2010.

[7] N. Simaan et al., "High dexterity snake-like robotic slaves for minimally invasive telesurgery of the upper airway," in Medical Image Computing and Computer-Assisted Intervention-MICCAI 2004. Springer, 2004, pp. 17-24.

[8] R. J. Murphy et al., "Design and kinematic characterization of a surgical manipulator with a focus on treating osteolysis," Robotica, vol. 32, no. 06, pp. 835-850, 2014.

[9] X. He et al., "Iris: Integrated robotic intraocular snake," in Robotics and Automation (ICRA), 2015 IEEE International Conference on. IEEE, 2015, pp. 1764-1769.

[10] Y.-J. Kim et al., "A stiffness-adjustable hyperredundant manipulator using a variable neutral-line mechanism for minimally invasive surgery," Robotics, IEEE Transactions on, vol. 30, no. 2, pp. 382395, 2014.

[11] M. H. Tawhai et al., "Generation of an anatomically based threedimensional model of the conducting airways," Annals of biomedical engineering, vol. 28, no. 7, pp. 793-802, 2000.

[12] J. J. Craig, Introduction to robotics: mechanics and control. Pearson Prentice Hall Upper Saddle River, 2005, vol. 3.

[13] J. Duchon, "Splines minimizing rotation-invariant semi-norms in sobolev spaces," in Constructive theory of functions of several variables. Springer, 1977, pp. 85-100. 